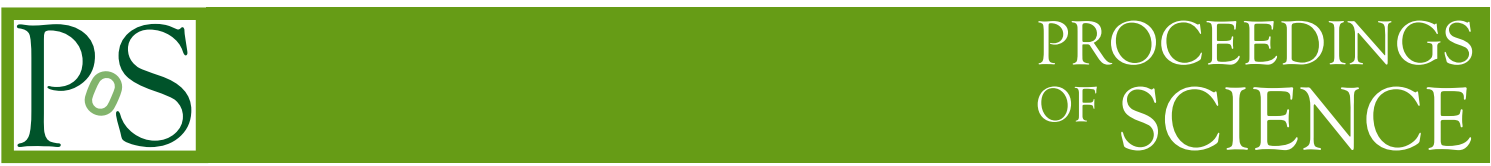

\title{
High angular radio observations of (U-)LIRGs
}

\author{
Antxon Alberdi*iand M. A. Pérez-Torres \\ Instituto de Astrofísica de Andalucía, IAA-CSIC \\ E-mail: antxon@iaa.es torres@iaa.es
}

\begin{abstract}
High angular resolution radio observations of the circumnuclear and nuclear regions of (Ultra)Luminous Infrared Galaxies ((U-)LIRGs) have revealed for some of them (Arp 299A, IC883, NGC 7469, between others) the presence of non-thermal compact components (Radio Supernovae and Supernova Remnants, constituting in some of the cases a supernova factory) in coexistence with a Low Luminosity AGN. These findings may suggest that both starbursts and AGN are frequently associated phenomena in mergers, favouring a composite AGN-starburst scenario. These findings may have an impact in the evolution of the AGN activity, being relevant for accreting models in the central regions of galaxies in the presence of recent bursts of star formation. The observations we show in this contribution make extensive use of the European VLBI Network $(\mathrm{EVN})$, a facility which was created and developped with the essential contribution of Richard T. Schilizzi.
\end{abstract}

Resolving the Sky - Radio Interferometry: Past, Present and Future -RTS2012

April 17-20, 2012

Manchester, $U K$

\footnotetext{
* Speaker.

† Other collaborators: C.Romero-Cañizales, R. Herrero-Illana, M. Bondi
} 


\section{Starburst Galaxies: the case of (U-)LIRGs}

Starbursts are intense periods of star formation within a galaxy, with typical star formation rates of around 10-100 $\mathrm{M}_{\odot} / \mathrm{yr}$ and lifetimes of $10^{7}-10^{8}$ years, short compared with the typical galaxy lifetime. The presence of a starburst is associated with a violent supply of gas to the central regions of the galaxy, a process which is usually triggered by merging and/or interaction between galaxies.

(Ultra-)Luminous Infrared Galaxies ((U-)LIRGs) are excellent candidates for starburst activity [12]. The high IR luminosity ( $\geq 10^{11} \mathrm{~L}_{\odot}$ for LIRGs and $\geq 10^{12} \mathrm{~L}_{\odot}$ for ULIRGs) is thought to be caused by dust grains that are heated by the presence of an AGN, a starburst or a combination of both. Indeed, it is possible to estimate the star formation rate for stars more massive than $\sim 5 M_{\odot}$ from the Far Infrared Luminosity, assuming that most of the energy in photons emitted by young stars is used for heating the dust. These massive stars dominate the overall stellar luminosity and end their lives as supernovae. Therefore, the detection of radio supernovae (RSNe) is a useful tool for the understanding of the physics of star formation, in particular in the dusty environments of the central regions of galaxies. The importance of (U-)LIRGs is based on the fact that a significant fraction of the Star Formation at high redshifts takes place in them [6]. Therefore, the detection of radio supernovae is crucial for revising the Core Collapse Supernova Rate (CCSN) both locally and at high redshift.

High resolution radio observations of nearby starburst galaxies have shown that its compact radio structure consists of a compact $\left(\leq 150 \mathrm{pc}\right.$ ), high surface brightness $\left(T_{b} \geq 10^{3} \mathrm{~K}\right)$ central radio source inmersed in a low surface brightness circumnuclear halo. The compact, centrally located radio emission in these starbursts might be generated by a point-like source (AGN), or by the combined effect of multiple radio supernovae and supernova remnants; on the other hand, it seems well established that the circumnuclear region of those objects host an ongoing burst of star formation. Radio interferometric observations of Luminous Infrared Galaxies (LIRGs) in our local Universe are a powerful tool to probe the dominant dust heating mechanism in their nuclear (using the EVN, VLBA) and circumnuclear (using eMERLIN, EVLA) regions.

\section{Some Scientific Cases}

In the following, we will discuss different scientific cases that we have studied in our group, making extensive use of the EVN and eMERLIN. These sources correspond to (U-)LIRGs at different stage mergers.

\subsection{Arp 299}

Arp 299 is an early stage merger at a distance of $45 \mathrm{Mpc}$ and a total luminosity of $6.7 \times 10^{11} \mathrm{~L} \odot$ [13]. When observed at radio wavelenghts, most of the luminosity is concentrated in two nuclei: Arp 299A -which emits $40 \%$ of the total luminosity, corresponding to a CCSN rate of $0.8 \mathrm{SN} / \mathrm{yr}-$ and Arp 299B -which emits 20\% of the total luminosity and a CCSN rate of $0.4 \mathrm{SN} / \mathrm{yr}-$.

We have observed the nuclear region of Arp 299A with the EVN at $6 \mathrm{~cm}$ during six epochs between April 2008 and November 2010 (Bondi et al.2012 [㚿]). We show in Fig 1, an image which is the result of the stacking of the 6 epochs of the eEVN images. In the central $150 \mathrm{pc}, 26$ 
compact sources are detected, showing a linear size smaller than $2 \mathrm{pc}$. We have obtained the first $5.0 \mathrm{GHz}$ radio light-curve (spanning $2.5 \mathrm{yr}$ ) of all compact components in the nuclear starburst of Arp 299-A. The properties (spectral index, variability) of all detected objects are consistent with them being a mixed population of CCSNe and SNRs. We have compared our images with previous observations at $8.4 \mathrm{GHz}$ performed by Neff et al.(2004) [7] and Ulvestad (2009) [15], identifying 8 new components; from those, we find clear evidence for at least two new CCSNe within our observing period, implying a lower limit to the CCSN rate of $\leq 0.80 \mathrm{SN} / \mathrm{yr}$, suggesting that the bulk of the current star formation in Arp 299-A is taking place in the innermost $150 \mathrm{pc}$ [2].

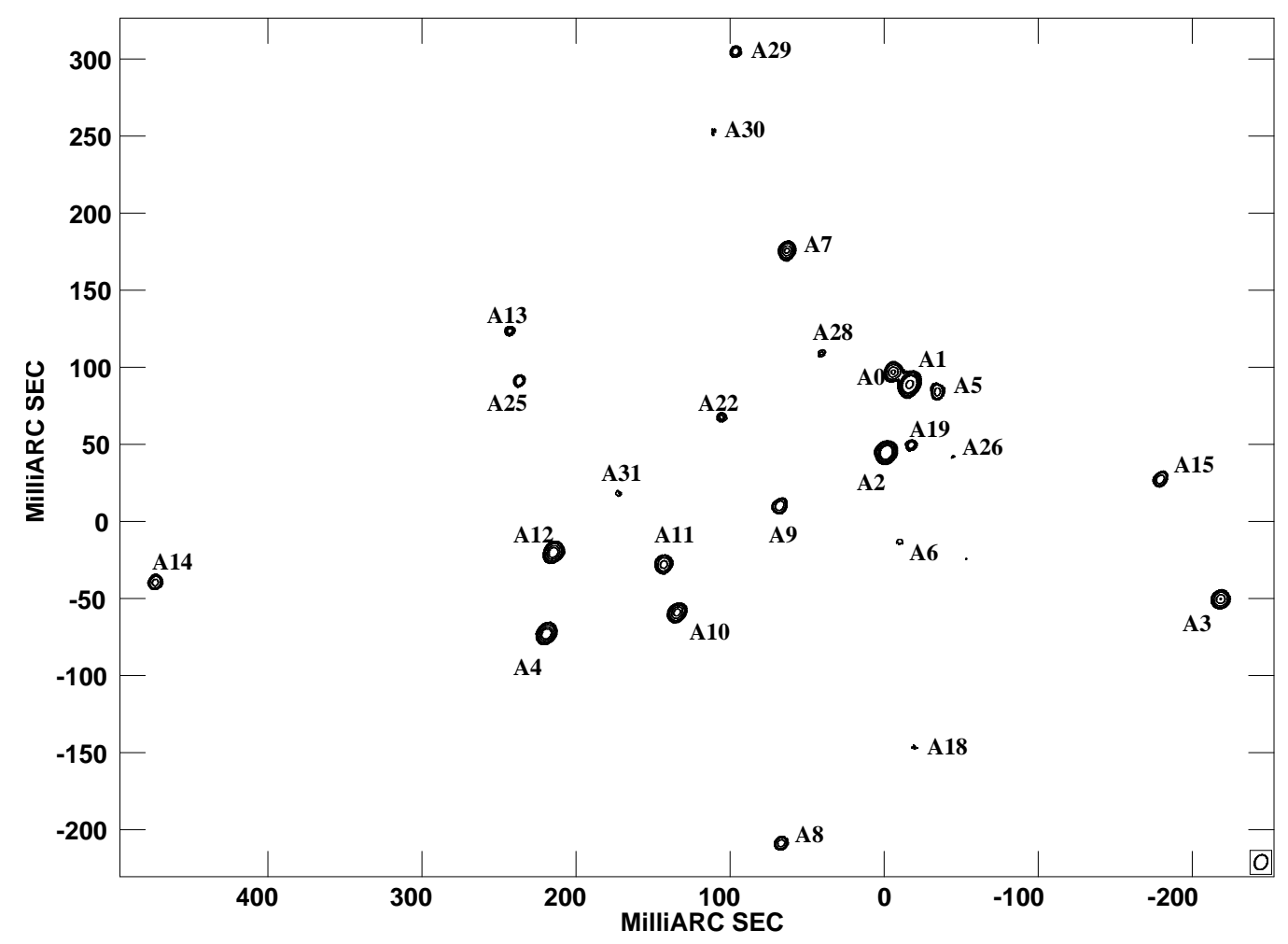

Figure 1: EVN image at 5.0 GHz of Arp299-A, obtained by stacking in the image plane six observing epochs between April 2008 and December 2010. This is the deepest image ever of the central 150 pc of Arp299-A at $5 \mathrm{GHz}$. The angular resolution is $10 \times 8$ mas, at a Position Angle of $25 \mathrm{deg}$, corresponding to a linear resolution of about $2 \mathrm{pc}$. The peak is $907 \mu \mathrm{Jy} / \mathrm{beam}$, and corresponds to the AGN candidate. Image taken from Bondi et al.(2012) [2].

We have performed quasi-simultaneous dual frequency (5.0 and $1.7 \mathrm{GHz})$ EVN observations of the inner 150 parsec of the nuclear region of Arp 299-A in order to understand their nature [10]. We have found that the brightest source of all the compact VLBI sources, the so called A1, previously detected at $5.0 \mathrm{GHz}$ and $8.4 \mathrm{GHz}$, has a flat spectrum between 1.7 and 5.0 GHz. Moreover, our $1.7 \mathrm{GHz}$ EVN image shows that A1 displays a core-jet structure, as traced by the diffuse, lowsurface brightness emission extending westwards from A1 towards A5 (see Fig. 2). Pérez-Torres et al. (2010) [10] have shown that the morphology, radio luminosity, spectral index and ratio of radio-to-X-ray emission of the A1-A5 region is consistent with a low-luminosity AGN (LLAGN). We concluded that the complex A1-A5 is the long-sought AGN in Arp 299-A, suggesting that 
Arp 299A has a composite AGN-Starburst nature. We also suggested that the presence of an AGN at a mere distance of 8 parsec from a stellar cluster could explain its low luminoity, suggesting that the star formation processes could be hindering the AGN activity.

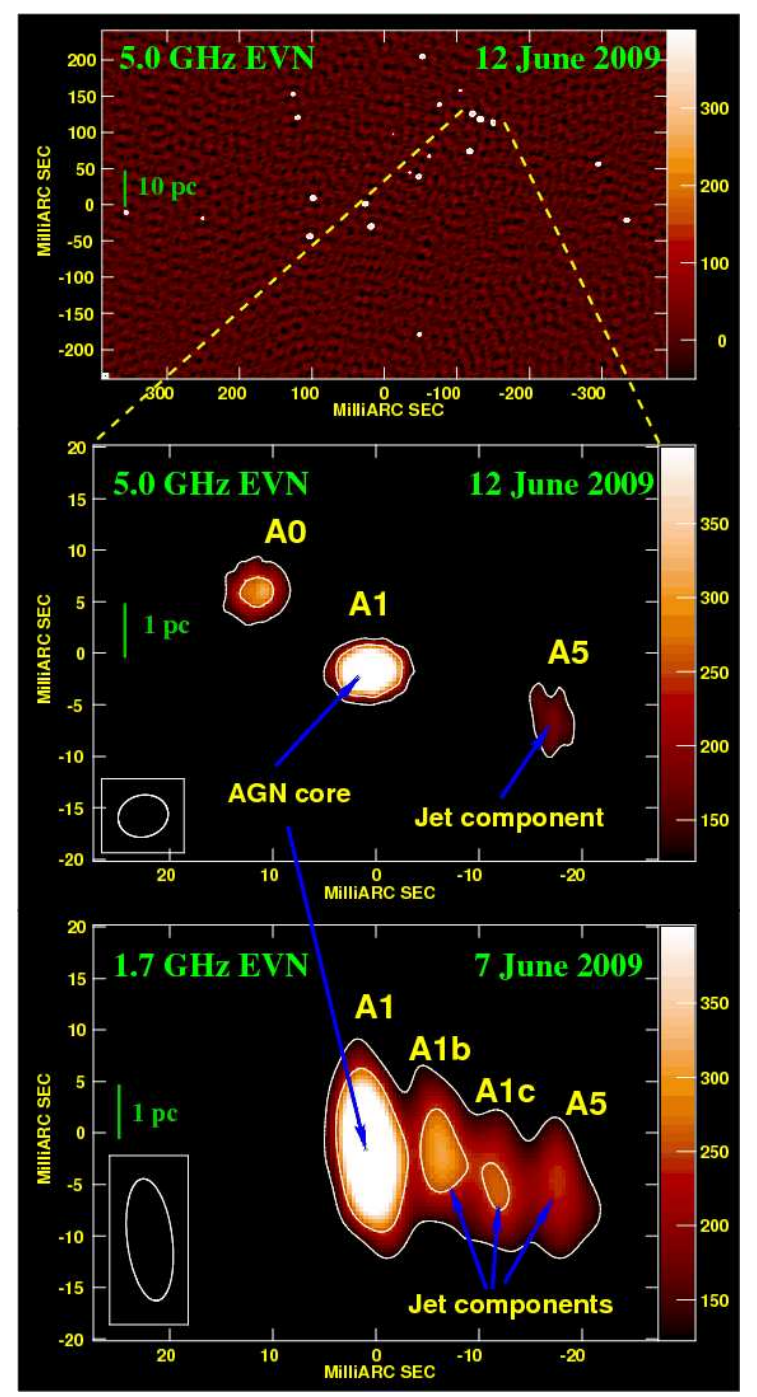

Figure 2: Top: $5.0 \mathrm{GHz}$ full EVN image of the central 150 parsec region of Arp 299-A, displaying a large number of bright, compact, nonthermal emitting sources. Middle and bottom: blow-ups of the inner 8 parsec of the nuclear region of Arp 299-A, as imaged with the full EVN at 1.7 and 5.0 GHz. The morphology, spectral index and luminosity of the A1-A5 region are very suggestive of a core-jet structure. Image taken from Pérez-Torres et al.(2010) [10].

\subsection{NGC 7469}

NGC 7469 is a well known barred spiral galaxy located at a distance of $70 \mathrm{Mpc}$, containing a luminous Seyfert 1 nucleus surrounded by a dusty starburst of about $1 \mathrm{kpc}$ in size. NGC 7469 is a very luminous infrared galaxy with $L_{F I R}=5 \times 10^{11} L_{\odot}$ were $2 / 3$ of the luminosity is emitted by the starburst [5]. Moreover, there is evidence for a $10^{7} M_{\odot}$ central black hole coming from reverberation 
mapping [8]. In 2000, we detected a new radio supernova, SN 2000ft, in the circumnuclear starburst of NGC 7469 (see Fig. 1), at a distance of 600 pc from the galaxy nucleus (Colina et al.2001 [3]). It is the first radio supernova ever detected in the circumnuclear ring of a Seyfert 1, luminous infrared galaxy. At a distance of $70 \mathrm{Mpc}, \mathrm{SN} 2000 \mathrm{ft}$ is one of the most luminous and distant RSNe ever detected. Although it has exploded in a radiation-dominated and highly magnetized environment, it behaves like a normal type-II radio supernova (Alberdi et al. 2006 [1]; Pérez-Torres et al. 2009 [9]).

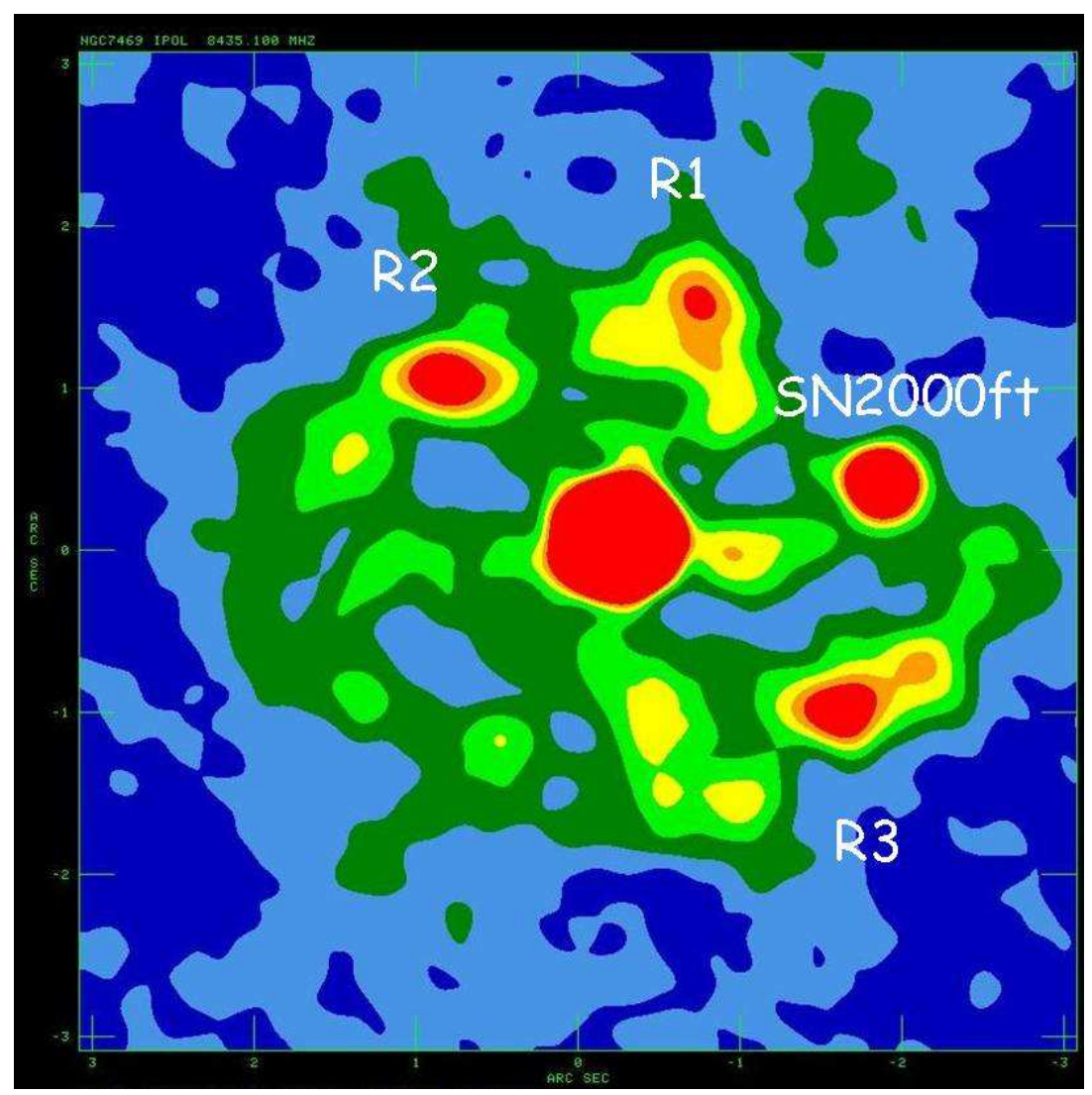

Figure 3: Total intensity image of NGC 7469 obtained at $8.4 \mathrm{GHZ}$ with the VLA in A-configuration, using all $u-v$ data for epochs 1998 through 2006. This is the deepest 8.4 GHZ image of NGC 7469 ever produced, with an off-source rms of $10 \mu \mathrm{Jy}_{\text {beam }}{ }^{-1}$. R1, R2 and R3 correspond to star-forming regions within the circumnuclear starburst of NGC 7469 (linear size of $\sim 1 \mathrm{kpc}$ ). SN 2000ft is a CCSNe that exploded at a distance of $600 \mathrm{pc}$ from the galaxy nucleus, within its circumnuclear starburst. Image taken from Pérez-Torres et al.(2009) [9].

We have performed quasi-simultaneous EVN observations of the nuclear regions of NGC 7469 at $6 \mathrm{~cm}$ and $18 \mathrm{~cm}$ (see Fig. 4). The image shows that the NGC 7469 nucleus consists of at least five compact sources located along an east-west line, with no evidence for diffuse emission or a jet-like structure connecting the compact sources between them. They are contained within an area of 150 mas (50pc) with a total flux density of $15 \mathrm{mJy}$ (at $18 \mathrm{~cm}$ ) and $10 \mathrm{mJy}$ (at $6 \mathrm{~cm}$ ). Some of the components are unresolved, while others show evidence for substructure and flux/structural time variability (for example, the westernmost one). 


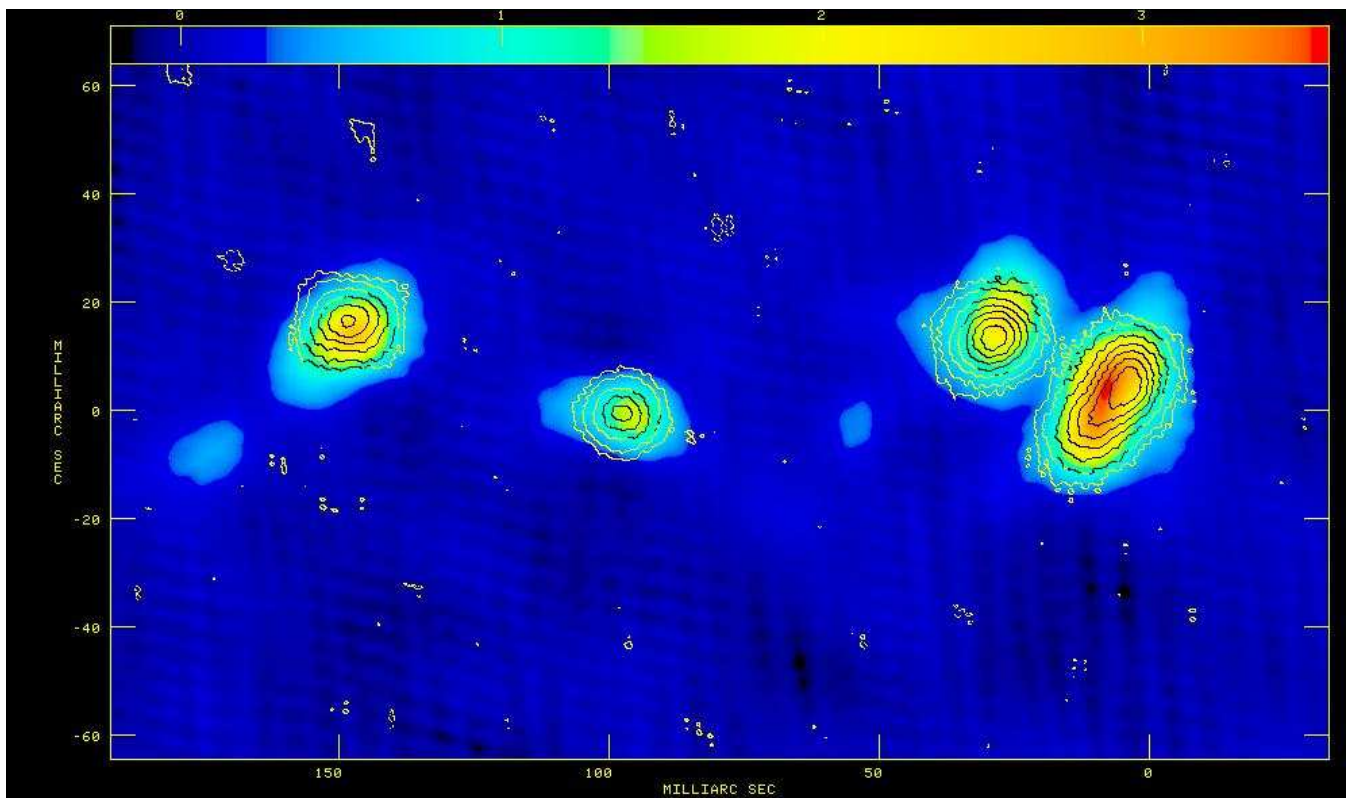

Figure 4: EVN images of the nuclear region of NGC 7469 (the linear size for the overall structure is of $\sim 50$ pc) obtained at 5.0 (colour) and $1.6 \mathrm{GHz}$ (contours).

It is still unclear whether these components are tracing i) the typical core-jet structure of an AGN, ii) compact starforming regions where individuals and/or clumps of radio supernovae are exploding, or iii) an starburst-AGN composite scenario: on the one hand, none of the components has a flat/inverted spectrum (all the nuclear components show steep spectra, with values between -1 and -0.3), which favours the interpretation that, at least, some of the components could be associated with young starforming regions -where core-collapse supernova are currently exploding-, and/or older regions, formed by chains of supernova remnants. We also note that all the VLBI sources are within an area of $50 \mathrm{pc}$, which is also the size of the nuclear starburst reported by Davies et al. (2004) [ 㺻; on the other hand, there is evidence for a $10^{7} M_{\odot}$ central black hole from reverberation mapping [8]. Moreover, the MERLIN and VLBI structures have a size and orientation consistent between them: the MERLIN core-jet structure [1] suggests that the westernmost component is the AGN core; in the VLBI images, the structural and flux-density variability suggest also that the core is located at the western side of the VLBI structure, although this result needs still to be confirmed via multi-epoch multi-frequency observations. Additionally, the supernova rate for NGC 7469 is a factor of 5 lower than for the archetype ULIRG Arp 220 (based on their infrared luminosities) and one would expect radio supernovae to be found in the starburst ring more than in the Seyfert 1 nucleus, which should be coincident with compact AGN emission. Due to the above reasons, we favour a composite Starburst-AGN scenario for the nuclear region of NGC 7469.

\subsection{IC 883}

IC 883 is a LIRG with an IR luminosity of about $4.7 \times 10^{11} \mathrm{~L}_{\odot}$ at a distance of $100 \mathrm{Mpc}$ [13]. This LIRG is an advanced stage member, probably the result of the merger of two disc galaxies (Smith et al. 1995) [14] as suggested by its associated tidal tails. 
We have imaged the circumnuclear and nuclear regions of IC 883 at radio frequencies with eMERLIN and the e-EVN, respectively (Romero-Cañizales et al. (2012) [11]; see Fig. 5). The e-MERLIN observations trace a double-sided structure, which is likely associated with a warped disc/ring. On the other hand, the nuclear region of IC 883 consists of at least four non-thermal components: three of these are transient sources within a 100 pc region -probably CCSNe candidatesand the fourth one is a long-lived, variable compact source, which is very likely an AGN. Thus, we are facing a situation with starburst and AGN driven activity in the innermost region of IC883: the AGN candidate powers the radio emission on both nuclear and circumnuclear scales; in addition, IC 883 displays very active star formation both within the innermost $100 \mathrm{pc}$ of its nucleus, and the circumnuclear regions. Romero-Cañizales et al. (2012) [11] have estimated a lower limit to the CCSN rate in the innermost nuclear regions of $\sim 0.6 \mathrm{SNe} / \mathrm{yr}$ based on the detection of three radio SNe above a luminosity threshold of $4 \times 10^{27} \mathrm{erg} / \mathrm{s} / \mathrm{Hz}$.

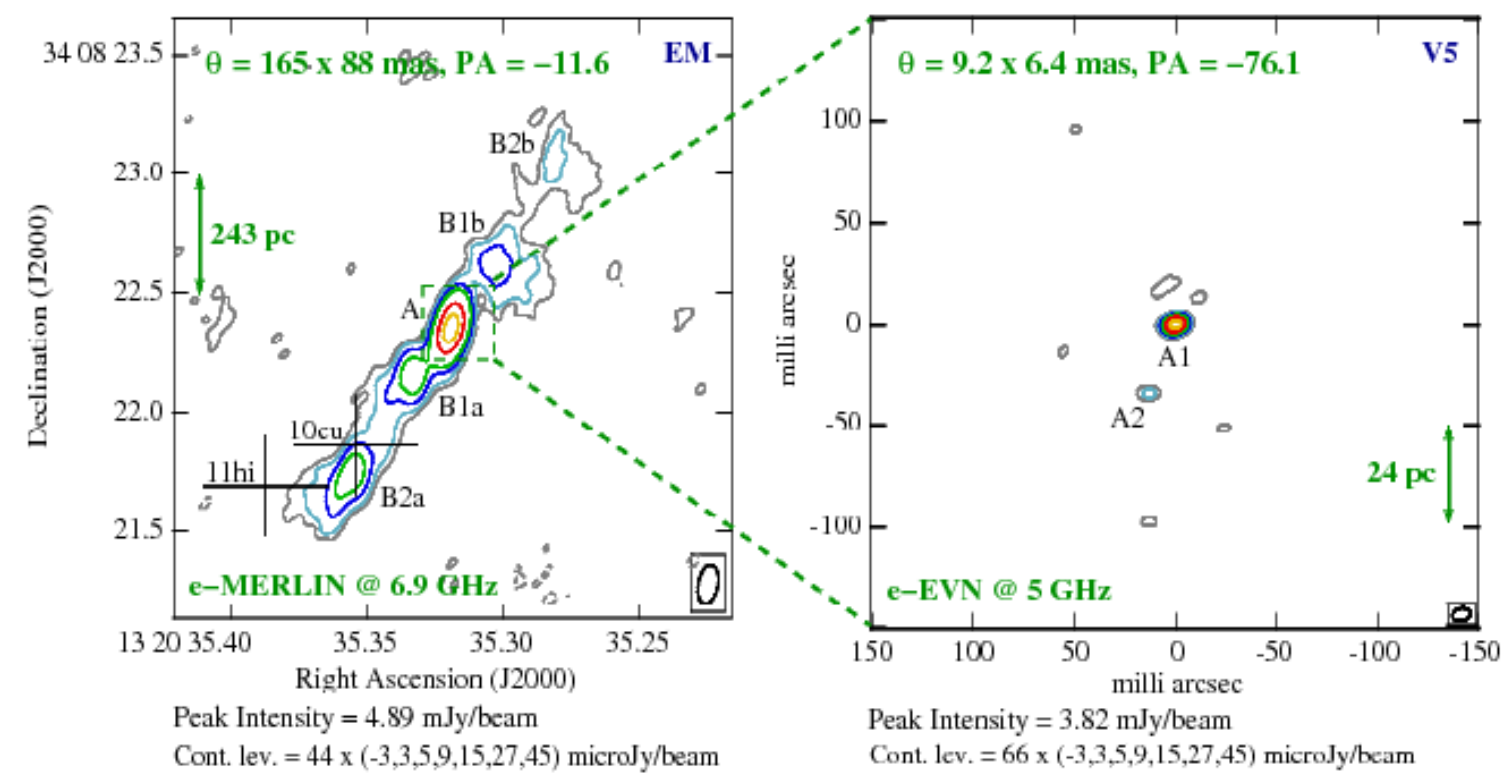

Figure 5: IC 883 contour images at $6.9 \mathrm{GHz}$ obtained with e-MERLIN (left) and at $5 \mathrm{GHz}$ with the e-EVN (right) performed in March 2011. The rms noise in the two images is 44 and $66 \mu \mathrm{Jy} / \mathrm{beam}$, respectively. Note that the brightest component at e-MERLIN scales is dominated by a compact source seen at mas-scales with the e-EVN. Image taken from Romero-Cañizales et al.(2012) [11].

\section{Summary}

Radio Observations of (U-)LIRGs at the highest angular resolution and sensitivity are extremely useful to discern starburst from AGN driven activity in the innermost regions. Our observations could also contribute to determine the core-collapse supernova rate and test the IMF for massive stars. In order to get meaningful results, high angular resolution radio searches of large samples -making extensive use of instruments like eMERLIN and the EVN- are needed. One of the co-authors of this contribution (MAPT) is co-PI of the eMERLIN Legacy Project "LIRGI - 
Luminous Infrared Galaxies Inventory": this project will allow to establish a phenomenological sequence and timescale for the evolution of nuclear starbursts in a statistically significant uniformly observed sample of (U-)LIRGs in the Local Universe. We have also been granted observations with the EVN for the LIRGI sample, which will provide additional information on the determination of the AGN and Starburst energy contribution in the nuclear regions of LIRGs.

\section{References}

[1] Alberdi A, Colina L, Torrelles J M et al.2006 ApJ 638938

[2] Bondi M, Pérez-Torres M A, Herrero-Illana R, Alberdi A 2012 A\&A 539 A134

[3] Colina L, Alberdi A, Torrelles J M, Panagia N and Wilson A S 2001 ApJ 553 L19

[4] Davies R L, Tacconi L J and Genzel R 2004 ApJ 602148

[5] Genzel R, Weitzel L and Tacconi-Garman L et al.1995 ApJ 444129

[6] Magnelli B, Elbaz D, Chary R R et al.2009, A\&A 49657

[7] Neff S, Ulvestad J S and Teng S H 2004, ApJ 611186

[8] Peterson B M and Wandel A 2000, ApJ 540 L13

[9] Pérez-Torres M A, Alberdi A, Colina L et al.2009 MNRAS 3991641

[10] Pérez-Torres M A, Alberdi A et al.2010 A\&A 519 L5

[11] Romero-Cañizales C, Pérez-Torres MA, Alberdi A et al.2012 A\&A 543, A72

[12] Sanders D B and Mirabel J F 1996 ARA\&A 34749

[13] Sanders D B, Mazzarella J M, Kim D. et al.2003 AJ 1261607

[14] Smith D A, Herter T, Haynes, M P et al.1995 ApJ 439623

[15] Ulvestad J S 2009 AJ 1381529 